\title{
G

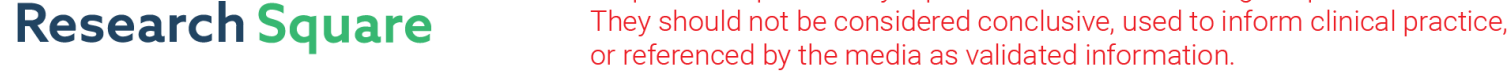 \\ Elderly patients of mild cognitive impairment exhibit altered profiles of the gut microbiota
}

\section{Qiong Pan}

Department of Obsterics and Gynecology, the Third Xiangya Hospital of Central South University,Changsha

\section{Ya-Qian Li}

Geratic Department, the Third Xiangya Hospital of Central South University, Changsha

\section{Ke Guo}

Department of Neurology, the Third Xiangya Hospital of Central South University, Changsha

\section{Min Xue}

Department of Obestetrics and Gynecology, the Third Xiangya Hospital of Central South University, Changsha

\section{Yu Gan}

Lin He's Academician Workstation of New Medicine and Clinical Translation at the Third Affiliated Hospital, Guangzhou Medical University, Guangzhou

\section{Kejian Wang ( $\nabla$ kejian-wang@foxmail.com )}

Guangzhou Medical University https://orcid.org/0000-0003-4221-2485

\section{Da-Bao Xu}

Department of Obstetrics and Gynecology, the Third Xiangya Hospital of Central South University, Changsha

Qiu-Yun Tu

Geratic Department, the Third Xiangya Hospital of Central South University, Changsha

\section{Research}

Keywords: mild cognitive impairment, Alzheimer's disease, gut microbiota, 16S rRNA quantitative microarray

Posted Date: September 18th, 2020

DOl: https://doi.org/10.21203/rs.3.rs-74865/v1

License: (c) (1) This work is licensed under a Creative Commons Attribution 4.0 International License. Read Full License 
Version of Record: A version of this preprint was published at Journal of Immunology Research on November 22nd, 2021. See the published version at https://doi.org/10.1155/2021/5578958. 


\section{Abstract \\ Background}

As the transitional state between normal aging and Alzheimer's disease (AD), mild cognitive impairment $(\mathrm{MCl})$ is characterized by cognitive decline greater than natural aging. While the association between $A D$ and gut microbiota has been reported in a number of studies, there is still very limited microbial research about $\mathrm{MCl}$.

\section{Methods}

Here we enrolled 48 participants, including $22 \mathrm{MCl}$ cases and 26 normal controls. Fecal samples were collected for $16 \mathrm{~S}$ rRNA quantitative arrays and bioinformatics analysis.

\section{Results}

Both Principal Coordinates Analysis (PCoA) and Non-metric Multidimensional scaling (NMDS) demonstrated that the microbial composition of $\mathrm{MCl}$ individuals deviated from the cluster of healthy controls. Multiple bacterial species were significantly increased (e.g., Staphylococcus intermedius) or decreased (e.g., Bacteroides salyersiae) in the samples from $\mathrm{MCl}$ group.

\section{Conclusion}

Therefore, the composition of gut microbiota differed between control subjects and $\mathrm{MCl}$ cases. Our study is the first to identify a series of $\mathrm{MCl}$ signature species in the gut microbiota, thus providing a new direction for future development of early diagnosis and probiotics regimen.

\section{Background}

Mild cognitive impairment $(\mathrm{MCl})$ is regarded as the transitional state between normal aging and Alzheimer's disease (AD) [1]. As a complicated syndrome, $\mathrm{MCl}$ is characterized by cognitive decline greater than natural aging, but without dramatic interference with daily life [2]. Epidemiological studies suggested that the prevalence of $\mathrm{MCl}$ is nearly $20 \%$ in the population older than 65 years [3]. In spite of seemingly normal status in some $\mathrm{MCl}$ patients, several clinical studies have found that most patients will eventually convert to $A D[4]$.

Emerging evidence indicated that disruption of the gut microbiome could undermine mental health. For clinical research, Zhuang et al. reported that a series bacteria taxa (e.g., Bacteroides, Ruminococcus and Actinobacteria) in AD patients were different from those in controls [5]. Vogt el al. identified significant differences in the abundance of Firmicutes (phylum), Bacteroidetes (phylum), and Bifidobacterium 
(genus) in the microbiota of AD cases. Moreover, there were correlations between the abundance of certain bacterial genera and biomarkers of $A D$ in cerebrospinal fluid [6]. In $A D$ animal models, gut microbiome has also been found to be correlated to impaired spatial learning and memory [7]. Li et al. documented similar changes in gut microbiome among $\mathrm{MCl}$ and $\mathrm{AD}$ cases [8]. However, there is still very limited evidence specifically showing the abnormalities of gut microbiota in $\mathrm{MCl}$ cases as compared to normal controls.

In the present study, potential alterations in the gut microbiota of cognitive impairment patients were investigated with 16S rRNA quantitative microarray, a novel high-throughput biotechnology for quantification of various bacteria taxa without conventional culture-based procedures $[9,10]$. Furthermore, we studied whether the microbiota composition is correlated to mental status parameters in cognitive impairment.

\section{Methods}

Study design and sample collection

Participants with mild cognitive impairment $(n=22)$ and control subjects $(n=26)$ were recruited from the Third Xiangya Hospital of Central South University. Mild cognitive impairment were diagnosed by the Geratic Department of the Third Xiangya Hospital of Central South University, China. This study was approved by the Ethics Committee of the Third Xiangya Hospital of Central South University and performed in accordance with relevant guidelines and regulations. Written informed consent was obtained prior to study from participants or a guardian on behalf of those participants who had impaired cognition. Exclusion criteria included: the use of antibiotics in the previous 6 months; large doses of probiotics consumed in the previous 3 months; current gastrointestinal disorders (e.g., chronic diarrhea, inflammatory bowel disease, infectious gastroenteritis, etc.); major gastrointestinal surgery in the past 3 years. The fresh stool samples were collected and saved in the sampling tubes with preservative solution. The tubes and preservative solution were provided by Halgen Ltd (China).

DNA extraction and labeling

Bacterial DNA was extracted from the stool samples using the Stool DNA Extraction Kit (Halgen, Ltd.) and by using the protocol as described in the product instruction manual. A universal primer pair was used to amplify the DNA the V1-V9 regions of the 16S rRNA gene. Approximately 20-30 ng of the extracted DNA was used in a $50 \mathrm{ul} \mathrm{PCR}$ reaction using the following cycling conditions: $94^{\circ} \mathrm{C}$ for $3 \mathrm{~min}$ for an initial denaturing step followed by $94^{\circ} \mathrm{C}$ for $30 \mathrm{~s}, 55^{\circ} \mathrm{C}$ for $30 \mathrm{~s}, 72{ }^{\circ} \mathrm{C}$ for $60 \mathrm{~s}$ for a total of 30 cycles followed by a final extension step of $72{ }^{\circ} \mathrm{C}$ for $3 \mathrm{~min}$. Agarose gel electrophoresis was run to check the success of PCR amplification. The PCR products were directly labeled without purification using a DNA labeled kit provided by Halgen Ltd. and processed for array hybridization according to the product instruction manual.

Microarray Hybridization 
Probes were selected from all the variable regions of bacterial 16S rRNA of bacteria. Each probe was designed to be about $40 \mathrm{bp}$ in length. The arrays were prepared by Halgen Ltd. using its proprietary technology. The hybridization mix typically contained $500 \mathrm{ng}$ of Cy5-labeled test sample DNA mixed with $50 \mathrm{ng}$ of a Cy3-labeled reference pool which serve to light up all the spots for accurate identification of spots and signal quantitation. The Cy3- and Cy5-labeled samples and hybridization buffer (Halgen Ltd.) were mixed together in a final volume of $150 \mu \mathrm{l}$, heated to $100{ }^{\circ} \mathrm{C}$ for $5 \mathrm{~min}$, and cooled on ice for $5 \mathrm{~min}$. All of these were put into a hybridization box and then hybridized in a hybridization oven with intermittent invention (Halgen Ltd.) for $3.5 \mathrm{~h}$ at $37^{\circ} \mathrm{C}$. Slides were washed in $2 \times$ SSC, $0.25 \%$ Triton X-100, $0.25 \%$ SDS, 1X Dye Protector (Halgen Ltd.) for $15 \mathrm{~min}$ at $63^{\circ} \mathrm{C}$, then rinsed in 1X Dye Protector till the slides clear of water droplets after immediate withdraw from the solution. Slides were scanned immediately using a dual-channel scanner.

\section{Data analysis}

All experiments involved co-hybridization of a Cy5-labeled test sample and a Cy3-labeled reference. We could determine the type of bacteria by the Cy5/Cy3 ratios of each probe. The relative abundance of each bacterial species was expected to be proportional to the mean of the Cy $5 /$ Cy3 ratios of the corresponding species-specific probes for that species. The image quantitation and subsequent analysis of relative abundance of bacteria were perform using a program provided by Halgen Ltd.

Alpha-diversity was calculated using and QIIME software [11] with default parameters. The differences of alpha-diversities between groups were calculated by Wilcoxon rank-sum test. PCoA and NMDS analyses were performed by QIIME modules and visualized by R packages (version 3.5.2). To detect statistical differences in beta diversity metrics between groups, we used permutational multivariate analysis of variance (PERMANOVA) in the vegan package in R. Linear Discriminant Analysis (LDA) Effect Size (LEfSe) [12] analysis was performed to analyze difference of bacterial species between groups. The pvalue for each species were calculated by Kruskal-Wallis test and Wilcoxon test. Unsupervised random forest clustering and receiver operating characteristic curve (ROC curve) proportional hazards statistics were also performed using R. To reduce the impact of overfitting, cross validation were performed by leave-one-out test in random forest clustering.

\section{Results}

Demographic data of study subjects

A total of 48 participants (22 MCl cases and 26 controls) were recruited from the Third Xiangya Hospital of Central South University. Gut microbiota was analyzed with fecal samples collected by clinicians (see Methods). As shown in Table 1, $\mathrm{MCl}$ and control groups did not differ with respect to female-to-male ratio, $\mathrm{BMI}$, or education, while only minor difference in average age was observed $(P=0.046)$. And there were significant differences $(P<0.01)$ between two groups in mental state and cognitive function as measured by MMSE score and index for Activities of Daily Living (ADL). 
Table 1

Baseline characteristics of study subjects.

\begin{tabular}{|c|c|c|c|}
\hline & $\begin{array}{l}\text { MCl Case }(n= \\
22)\end{array}$ & $\begin{array}{l}\text { Control }(n= \\
26)\end{array}$ & $P$-value \\
\hline Age $(y r s$, mean $\pm S D)$ & $71.45 \pm 8.03$ & $67.31 \pm 5.27$ & 0.046 \\
\hline Sex (Female/Male) & $14 / 8$ & $19 / 7$ & 0.543 \\
\hline $\mathrm{BMI}\left(\mathrm{kg} / \mathrm{m}^{2}\right.$, mean $\left.\pm \mathrm{SD}\right)$ & $23.78 \pm 3.98$ & $22.05 \pm 5.10$ & 0.194 \\
\hline Education & & & 0.87 \\
\hline Illiteracy & 5 & 7 & \\
\hline Elementary School & 11 & 11 & \\
\hline High School & 6 & 8 & \\
\hline MMSE Score $($ mean \pm SD) & $15.55 \pm 4.50$ & $23.96 \pm 2.84$ & $\begin{array}{l}7.81 \times 10 \\
9\end{array}$ \\
\hline $\begin{array}{l}\text { Hamilton Depression Rating Scale (HAM-D, mean } \\
\pm S D)\end{array}$ & $5.09 \pm 4.85$ & $4.08 \pm 3.63$ & 0.424 \\
\hline $\begin{array}{l}\text { Index for Activities of Daily Living (ADL, mean } \pm \\
\text { SD) }\end{array}$ & $25.95 \pm 8.14$ & $21.04 \pm 1.28$ & 0.01 \\
\hline
\end{tabular}

$\mathrm{MCl}$ cases harbor an altered gut microbiota

Compositional analysis indicated the presence of 19 phyla across all samples, but only 7 of them were above the mean value of $1 \%$ of the total abundance (Fig. 1A). The relative abundance of Bacteroidetes was found to be lower in $\mathrm{MCl}$ group than in control group. On the other hand, Fusobacteria were significantly more abundant in $\mathrm{MCl}$ cases than in controls.

The analysis of a-diversity (Fig. 2) included calculation of Chao, ACE, Shannon, and Simpson indices, but no significant difference between $\mathrm{MCl}$ and control groups was detected (P-value $>0.05$ ). The analysis of $\beta$-diversity, including Principal Coordinate Analysis (PCoA) and Non-metric Multidimensional Scaling (NMDS), demonstrated that the gut microbiota profiles of $\mathrm{MCl}$ cases clustered apart from those of control subjects (Fig. 3, PERMANOVA P-value $=\mathrm{P}=0.048$ ). Such separation indicated that $\mathrm{MCl}$-related changes may occur in certain bacterial taxa.

Association between bacterial abundance and cognitive status

Given the MCl-related alterations in gut microbiota, an in-depth analysis was performed by using linear discriminant effect size (LEfSe) algorithm (see Methods section). A series of bacterial taxa were identified for differential abundance between $\mathrm{MCl}$ cases and normal controls (Fig. 4A, Table S1). Particularly at species level, the 16S rRNA microarray revealed significant enrichment of 9 species (e.g., Staphylococcus 
intermedius, Fig. 4B) and attenuation of 25 species (e.g., Bacteroides salyersiae, Fig. 4C) among MCl cases.

\section{Discussion}

$\mathrm{MCl}$ has important implications for the health of the elderly, since those with history of $\mathrm{MCl}$ are more likely to develop $A D$ in the long term $[13,14]$. A number of studies have provided compelling evidence that dysbiosis plays an important role in the pathogenesis of $A D$ and many other neurodegenerative diseases [15]. However, the research on gut microbiome exposed to $\mathrm{MCl}$ is limited. In the present study, we initiatively examined the gut dysbiosis in $\mathrm{MCl}$ cases.

In our results, the relationship between bacterial taxonomic profile and $\mathrm{MCl}$ was not characterized by altered a-diversity. But a separation between $\mathrm{MCl}$ and control groups can be visualized in $\beta$-diversity analysis, suggesting abnormal depletion of certain bacterial taxa. In particular, the reduction of Bacteroides salyersiae and Bacteroides gallinarum in $\mathrm{MCl}$ cases was in line with previous research on $\mathrm{AD}$. Zhuang et al. reported depletion of Bacteroides genus in AD but could not specify the depleted species by conventional 16S rRNA sequencing [5]. In fact, Bacteroides fragilis, another species of Bacteroides genus was reported to be decreased in patients with cognitive impairment and brain amyloidosis [16]. Our finding further corroborated the relevance of Bacteroides genus in gut microbiota to neurodegenerative diseases and provided 2 more species, which can be used as potential biomarkers for early detection of $\mathrm{MCl} / \mathrm{AD}$ risks [17].

On the other hand, the enrichment of certain taxa in $\mathrm{MCl}$ subjects were also found to be related to neurodegeneration. For example, Staphylococcus intermedius and Staphylococcus lentus of Staphylococcus genus exhibited significantly higher abundance in $\mathrm{MCl}$ group than in control group.

A series of studies have suggested that Staphylococcus are involved in the generation of extracellular amyloid fibers [18] through multiple mechanisms, including regulation of phenol soluble modulins (PSMs). PSMs produced by Staphylococcus have been documented to form amyloid fibers in biofilms [19]. While most published evidence pointed to the relevance of Staphylococcus aureus, our results identified two other species in the same genus, thus expanding the scope of investigating the role played by in Staphylococcus in neurodegeneration.

Our study is notable for certain technique advantages. First, among numerous studies on Alzheimer's disease, the present research initiatively investigated $\mathrm{MCl}$ as a separate phenotype, thus providing unique insights into the progression from $\mathrm{MCl}$ to $\mathrm{AD}$. Second, unlike 16S rRNA sequencing that only provide genus-level data [20], the $16 \mathrm{~S}$ quantitative microarray technology enabled us to scrutinize MCl-related alterations in gut microbiome at species level. This benefic would not only shed light on the role played by brain-gut axis in the process of neurodegeneration, but also promote the development of more precise diagnostic methods for $\mathrm{MCl}$ based on gut microbiota signatures. 
In the meantime, several limitations of our results should be taken into consideration. For example, due to the complex process of participant enrollment and strict exclusion criteria, the size of our sample was restricted. The relatively low sample size does not allow to exclude the $\beta$-error when statistical significance is not reached. This could partially explain the discovery of some differentially abundant taxa in our $\mathrm{MCl}$ samples but not in previously published results on AD patients. In addition, since all the participants were recruited from the same hospital, potential regional variations of gut microbiota could not be evaluated. Our further study is aimed at a multi-center clinical research with larger the sample size to thoroughly investigate the gut microbiota among $\mathrm{MCl}$ subjects across different regions.

In conclusion, the present study provided new evidence on the abnormalities of gut microbiota in mild cognitive impairment cases as compared to controls subjects. Our results can guide the development of microbiota-based diagnosis for detecting early risks of mild cognitive impairment and subsequent Alzheimer's disease. And the newfound bacteria with alterations in mild cognitive impairment may provide clues for probiotics regimen that alleviate age-associated cognitive decline.

\section{Declarations}

\section{Ethics approval and consent to participate}

The written informed consent was obtained from all participants. The Ethics Committee of the Third Xiangya Hospital of Central South University approved the study.

\section{Consent for publication}

Not applicable.

\section{Availability of data and materials}

The datasets used and/or analyzed during the current study are available from the corresponding author on reasonable request.

\section{Competing interests}

None of the other authors have competing interests.

\section{Funding}

National Natural Science Foundation of China (81671398)

\section{Authors' Contributions}


QP, KW and QT designed the study; QP, YL and KG collected samples and conducted experiments; QP, MX, YG, KW, DX and QT wrote the manuscript.

\section{Acknowledgements}

This study is supported by the National Natural Science Foundation of China (81671398), the Natural Science Foundation of Hunan Province, China (2019JJ50912) and the Research Fund for Lin He's Academician Workstation of New Medicine and Clinical Translation.

\section{References}

1. Petersen RC, et al. Mild cognitive impairment: clinical characterization and outcome. Arch Neurol. 1999;56(3):303-8.

2. Gauthier S, et al. Mild cognitive impairment Lancet. 2006;367(9518):1262-70.

3. Goldman WP, Morris JC. Evidence that age-associated memory impairment is not a normal variant of aging. Alzheimer Dis Assoc Disord. 2001;15(2):72-9.

4. DeCarli C. Mild cognitive impairment: prevalence, prognosis, aetiology, and treatment. Lancet Neurol. 2003;2(1):15-21.

5. Zhuang ZQ, et al. Gut Microbiota is Altered in Patients with Alzheimer's Disease. J Alzheimers Dis. 2018;63(4):1337-46.

6. Vogt NM, et al. Gut microbiome alterations in Alzheimer's disease. Sci Rep. 2017;7(1):13537.

7. Shen L, Liu L, Ji HF. Alzheimer's Disease Histological and Behavioral Manifestations in Transgenic Mice Correlate with Specific Gut Microbiome State. J Alzheimers Dis. 2017;56(1):385-90.

8. Li B, et al. Mild cognitive impairment has similar alterations as Alzheimer's disease in gut microbiota. Alzheimers Dement. 2019;15(10):1357-66.

9. Cai WW, et al. An SSLP marker-anchored BAC framework map of the mouse genome. Nat Genet. 2001;29(2):133-4.

10. Cai WW, et al. Genome-wide detection of chromosomal imbalances in tumors using BAC microarrays. Nat Biotechnol. 2002;20(4):393-6.

11. Kuczynski J, et al., Using QIIME to analyze 16S rRNA gene sequences from microbial communities. Curr Protoc Bioinformatics, 2011. Chapter 10: p. Unit 107.

12. Segata N, et al. Metagenomic biomarker discovery and explanation. Genome Biol. 2011;12(6):R60.

13. Ardekani BA, et al. Prediction of Incipient Alzheimer's Disease Dementia in Patients with Mild Cognitive Impairment. J Alzheimers Dis. 2017;55(1):269-81.

14. Herukka SK, et al. Recommendations for cerebrospinal fluid Alzheimer's disease biomarkers in the diagnostic evaluation of mild cognitive impairment. Alzheimers Dement. 2017;13(3):285-95. 
15. Bostanciklioglu M. The role of gut microbiota in pathogenesis of Alzheimer's disease. J Appl Microbiol. 2019;127(4):954-67.

16. Cattaneo A, et al. Association of brain amyloidosis with pro-inflammatory gut bacterial taxa and peripheral inflammation markers in cognitively impaired elderly. Neurobiol Aging. 2017;49:60-8.

17. Barber RC. Biomarkers for early detection of Alzheimer disease. J Am Osteopath Assoc. 2010;110(9 Suppl 8):S10-5.

18. Schwartz K, Boles BR. Microbial amyloids-functions and interactions within the host. Curr Opin Microbiol. 2013;16(1):93-9.

19. Schwartz K, et al. Functional amyloids composed of phenol soluble modulins stabilize Staphylococcus aureus biofilms. PLoS Pathog. 2012;8(6):e1002744.

20. Wang Q, et al. Naive Bayesian classifier for rapid assignment of rRNA sequences into the new bacterial taxonomy. Appl Environ Microbiol. 2007;73(16):5261-7.

\section{Figures}

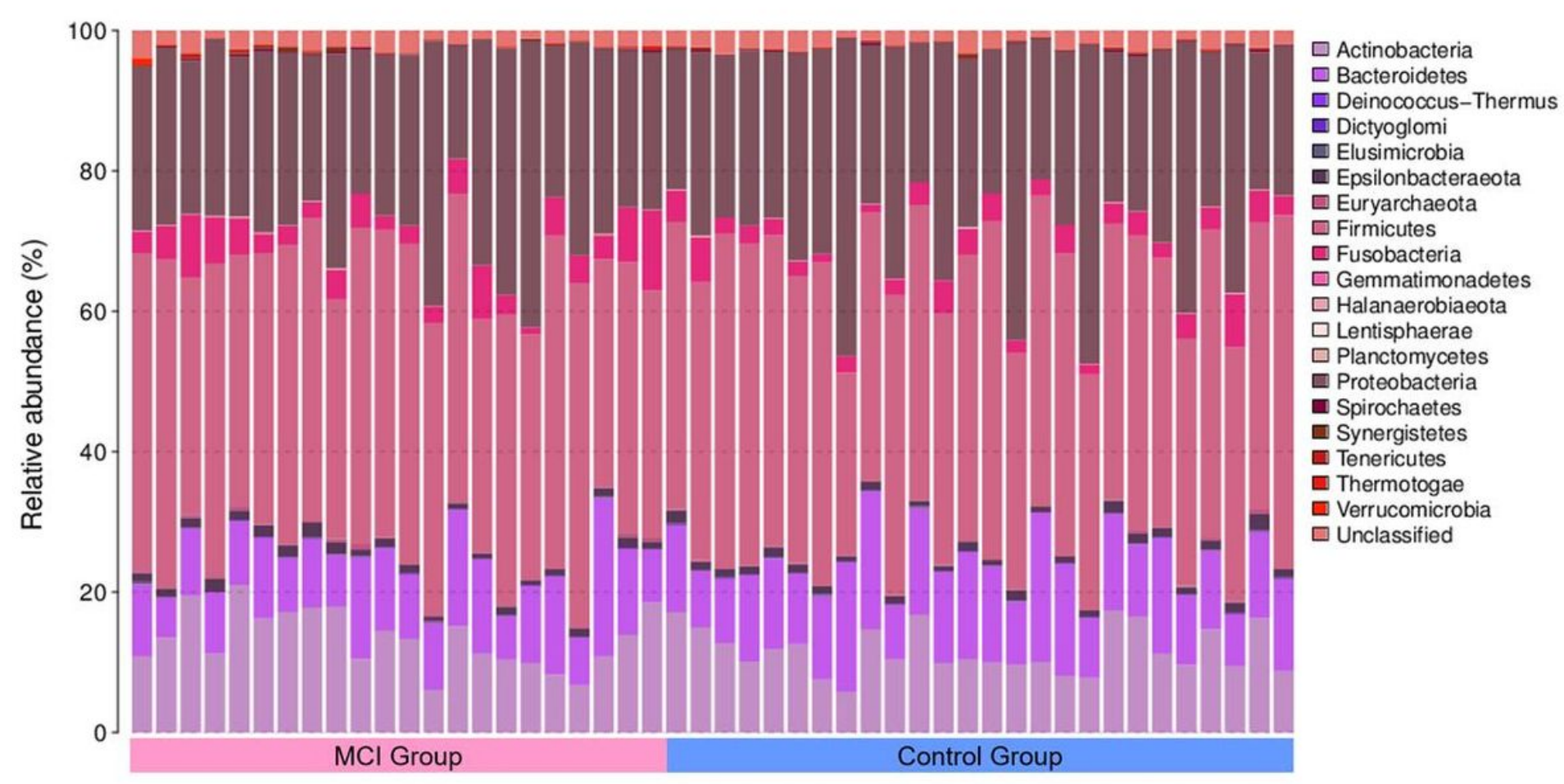

Figure 1

Proportion of the different phyla (represented with different colors) detected in $\mathrm{MCl}$ cases and control subjects. 
A

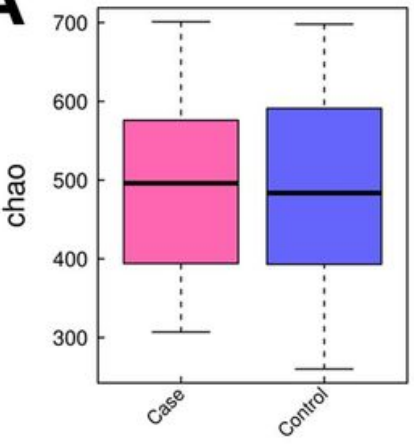

B

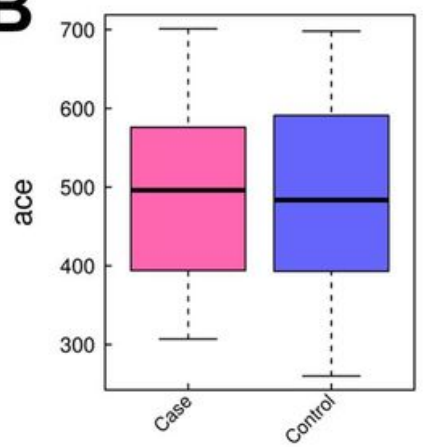

C

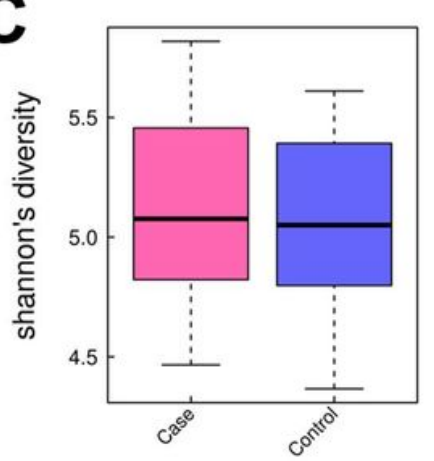

D

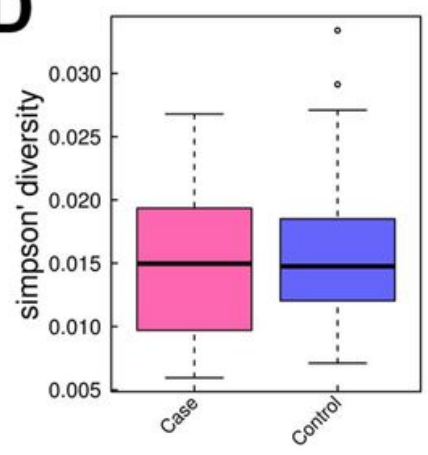

Figure 2

Alpha diversity in $\mathrm{MCl}$ and control groups as represented by Chao index (A), ACE index (B), Shannon diversity (C) and Simpson diversity (D).
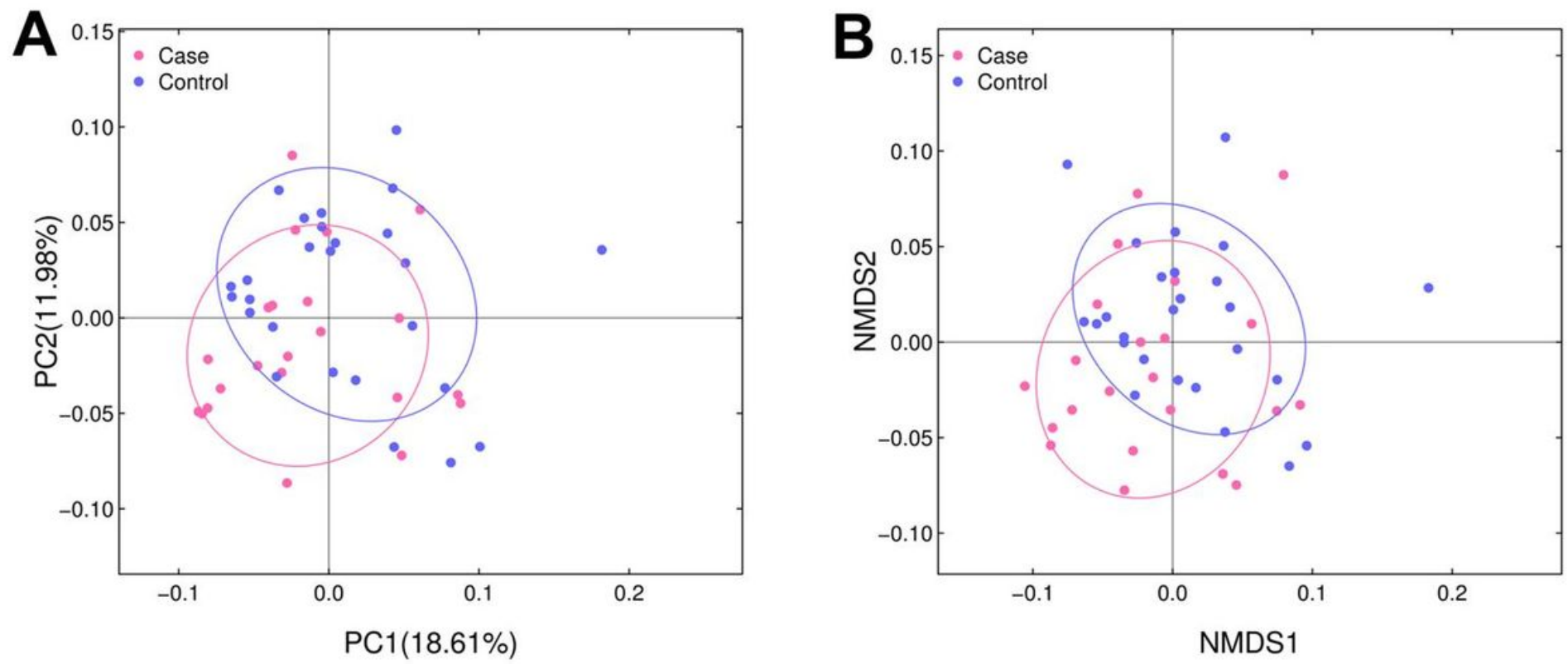

Figure 3

Analysis of $\beta$-diversity based on PCoA (A) and NMDS (B) with weighted UniFrac distance. $\mathrm{MCl}$ cases and control subjects are denoted with pink and blue nodes, respectively. 
$A=\cdots$

as Gordonibacter E: Lactobacilus m: Lachnospira si fusobacteris

b: Bacteroides h:Streptococtaceae n; Phacolarctobactenium t: Methylobacterium

esacteroidacese it Lactobacilales o: Leptotrichis us Bejesinckistese

d:Alloprevotela i:Anoxynatronom pileptotrichiacese viuncultured

e: Prevotellaceas k: Pseudoramibacter q: Fusobacteriales w: tysobacter

f: Baxteroidetes I: Erakiela rifusobacteria

B
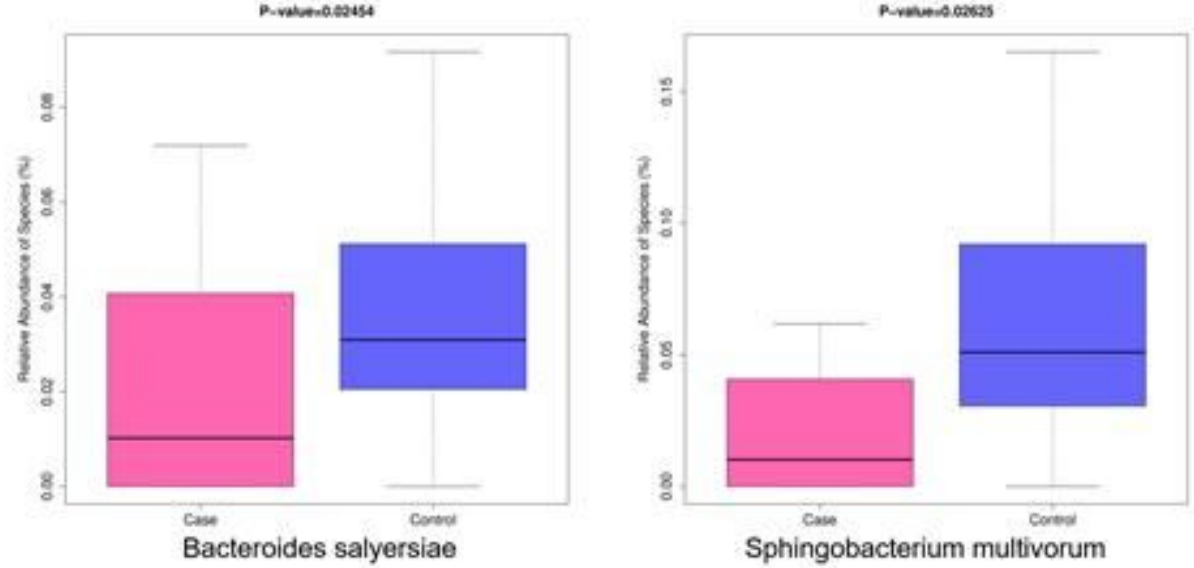

C
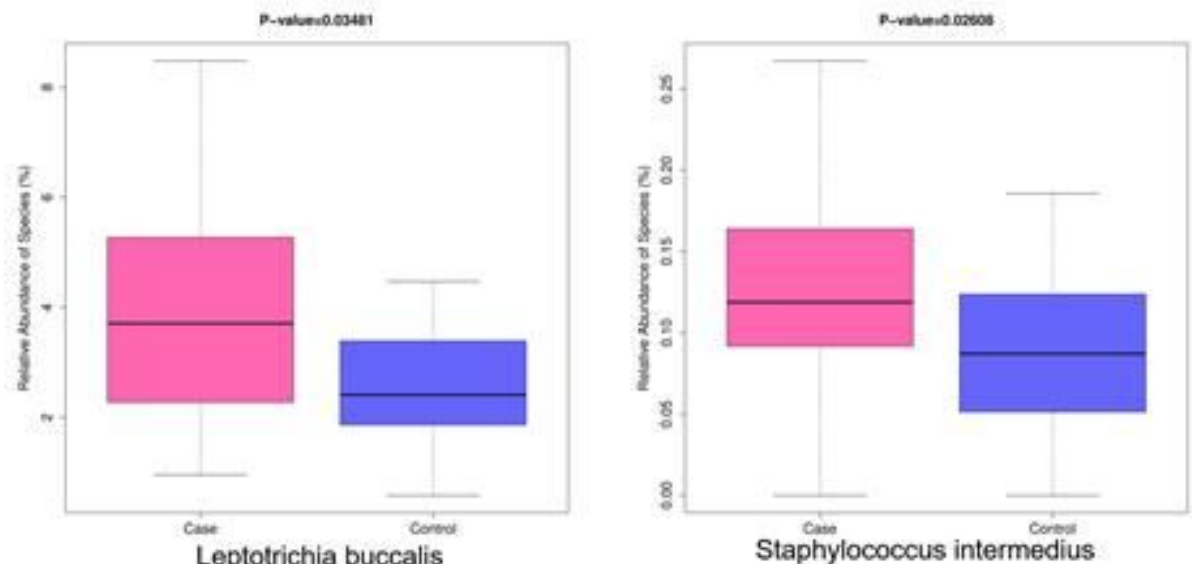

\section{Figure 4}

Bacterial taxa differentially represented in samples from $\mathrm{MCl}$ cases compared to control subjects. (A) Cladograms generated by LEfSe software showing the differences. Nodes in pink and blue indicate taxa that were enriched in $\mathrm{MCl}$ group and control group, respectively. (B) Representative species with decreased abundance in $\mathrm{MCl}$ group. (C) Representative species with increased abundance in $\mathrm{MCl}$ group.

\section{Supplementary Files}

This is a list of supplementary files associated with this preprint. Click to download. 
- TableS1.docx

- Tables1.docx

Page 13/13 